\title{
CURVE SELECTION LEMMA FOR SEMIANALYTIC SETS AND CONJUGACY CLASSES OF FINITE ORDER IN LIE GROUPS
}

\author{
JINPENG AN AND ZHENGDONG WANG
}

\begin{abstract}
Using a strong version of the Curve Selection Lemma for real semianalytic sets, we prove that for an arbitrary connected Lie group $G$, each connected component of the set $E_{n}(G)$ of all elements of order $n$ in $G$ is a conjugacy class in $G$. In particular, all conjugacy classes of finite order in $G$ are closed. Some properties of connected components of $E_{n}(G)$ are also given.
\end{abstract}

\section{INTRODUCTION}

The interest of real semianalytic geometry (or, more generally, real subanalytic geometry) lies not only on its own right but also on its applications in many fields, such as calculus of variations and control theory. In this paper we give an application of real semianalytic geometry to the problem of the closedness of conjugacy classes of finite order in Lie groups. The availability of real semianalytic geometry owes to the fact that each Lie group admits a unique real analytic structure such that multiplication, inversion, the exponential map, and homomorphisms are real analytic (see, for example, Varadarajan [4, Section 2.11). Using a strong version of the Curve Selection Lemma for real semianalytic sets, we will prove the following conclusion.

Theorem 1.1. Let $G$ be a connected Lie group, $n$ a positive integer. Denote $E_{n}(G)=\left\{g \in G \mid g^{n}=e\right\}$. Then each connected component of $E_{n}(G)$ is a conjugacy class of $G$.

Since $E_{n}(G)$ is a closed subset of $G$, its connected components are closed in $G$. So Theorem 1.1 implies immediately that all conjugacy classes of order $n$ in $G$ are closed.

It is well known that semisimple conjugacy classes in algebraic groups are Zariski closed (see, for example, Humphreys [2]), and a conjugacy class of finite order in an algebraic group is necessary semisimple. So Theorem 1.1 may be viewed as a generalization of this result to Lie groups.

In Section 2, we will present the version of the Curve Selection Lemma that will be needed. Based on it, the proof of Theorem 1.1 will be given in Section 3.

As corollaries of Theorem 1.1 some properties of the connected components of the set $E_{n}(G)$ will be given in Section 4. Denote the set of all connected components of $E_{n}(G)$ by $\mathcal{E}_{n}(G)$. The most fundamental property of $\mathcal{E}_{n}(G)$ is that each element of $\mathcal{E}_{n}(G)$ is a closed submanifold of $G$, which is an obvious corollary of Theorem 1.1. Of course, different elements of $\mathcal{E}_{n}(G)$ may have different dimensions which,

2000 Mathematics Subject Classification. Primary 14P15; Secondary 22E15.

Key words and phrases. semianalytic set, Curve Selection Lemma, Lie group, conjugacy class.

This work is supported by the 973 Project Foundation of China ( $\sharp$ TG1999075102). 
as the dimensions of conjugacy classes, can be easily computed. Another corollary is that $\mathcal{E}_{n}(G) \cap \mathcal{E}_{m}(G)=\mathcal{E}_{(n, m)}(G)$ for any positive integers $n, m$, where $(n, m)$ is the greatest common divisor of $n$ and $m$. The following property will also be proved. Let $K$ be a maximal compact subgroup of $G$, and let $i: K \rightarrow G$ be the inclusion map. We will show that the natural induced map $i_{*}: \mathcal{E}_{n}(K) \rightarrow \mathcal{E}_{n}(G)$ is a bijection. In particular, $\mathcal{E}_{n}(G)$ is a finite set, and the intersection of each connected component of $E_{n}(G)$ with $K$ is a connected component of $E_{n}(K)$.

\section{A version of the Curve Selection lemma for semianalytic sets}

In this section we present a strong version of the Curve Selection Lemma for real semianalytic sets, which will be needed in the proof of Theorem 1.1 For a real semianalytic set $X$, the sets of regular points and singular points in $X$ are denoted by $r(X)$ and $s(X)$, respectively. The following Wing Lemma is a key ingredient.

Proposition 2.1. (Wing Lemma [3, 15) Let $m<n$ be non-negative integers, and identify $\mathbb{R}^{m}$ with $\left\{\left(x_{1}, \cdots, x_{n}\right) \in \mathbb{R}^{n} \mid x_{m+1}=\cdots=x_{n}=0\right\}$. Let $U \subset \mathbb{R}^{n}$ be an open neighborhood of $0 \in \mathbb{R}^{n}$, and let $X \subset U \cap\left(\mathbb{R}^{n}-\mathbb{R}^{m}\right)$ be a semianalytic set. Suppose $U \cap \mathbb{R}^{m} \subset \bar{X}$. Then there exist semianalytic sets $W, Z$ of $\mathbb{R}^{n}$ such that

(i) $W \subset X, U \cap \mathbb{R}^{m} \subset \bar{W}, \operatorname{dim}(W)=m+1$;

(ii) $Z \subset U \cap \mathbb{R}^{m}, Z$ is closed and nowhere dense in $U \cap \mathbb{R}^{m}, \operatorname{dim}(Z)<m$;

(iii) For each $p \in\left(U \cap \mathbb{R}^{m}\right)-Z$, there is an open neighbourhood $V \subset U$ of $p$ in $\mathbb{R}^{n}$ such that for each connected component $W_{r}$ of $W \cap V, W_{r} \cup\left(V \cap \mathbb{R}^{m}\right)$ is an $(m+1)$-dimensional $C^{1}$ submanifold of $\mathbb{R}^{n}$ with boundary $V \cap \mathbb{R}^{m}$, and there exists a $C^{1}$ function $y^{r}: W_{r} \cup\left(V \cap \mathbb{R}^{m}\right) \rightarrow \mathbb{R}$ such that $\left(x_{1}, \cdots, x_{m}, y^{r}\right)$ forms a $C^{1}$ coordinate system of $W_{r} \cup\left(V \cap \mathbb{R}^{m}\right)$.

Proposition 2.2. (Curve Selection Lemma) Let $X$ be a semianalytic set in a real analytic manifold $M$. Suppose $s(X) \neq \emptyset$. Then there exists a semianalytic set $Z \subset s(X)$ which is closed and nowhere dense in $s(X)$ with $\operatorname{dim}(Z)<\operatorname{dim}(s(X))$ such that for each $p \in s(X)-Z$, there exists a $C^{1}$ curve $\gamma:[0,1) \rightarrow M$ such that $\gamma(0)=p, \gamma((0,1)) \subset r(X)$, and $\gamma^{\prime}(0)$ does not belong to the tangent space $T_{p} N$ of any analytic immersed submanifold $N$ of $M$ with $p \in N \subset s(X)$.

Proof. Choose a locally finite family of analytic coordinate charts $\left\{\left(U^{i}, x_{1}^{i}, \cdots, x_{n}^{i}\right) \mid i \in\right.$ $I\}$ such that $r(s(X)) \subset \bigcup_{i \in I} U^{i}$ and $U^{i} \cap s(X)=\left\{p \in U^{i} \mid x_{m_{i}+1}^{i}(p)=\cdots x_{n}^{i}(p)=\right.$ $0\}$, where $n=\operatorname{dim}(M), m_{i} \leq \operatorname{dim}(s(X))$ (such a family of charts always exists since $r(s(X))$ is open in $s(X))$. By the Wing Lemma, for each $i \in I$, there exists a semianalytic set $Z^{i} \subset U^{i} \cap s(X)$ which is closed and nowhere dense in $U^{i} \cap s(X)$ with $\operatorname{dim}\left(Z^{i}\right)<m_{i}$ such that for each $p \in\left(U^{i} \cap s(X)\right)-Z^{i}$, there exists a $C^{1}$ curve $\gamma:[0,1) \rightarrow U^{i}$ such that $\gamma(0)=p, \gamma((0,1)) \subset r(X) \cap U^{i}$, and $\gamma^{\prime}(0) \notin T_{p}(s(X))$ (note that $T_{p}(s(X))$ is well-defined since $\left.p \in r(s(X))\right)$. In fact, the $y^{r}$-axis in Proposition 2.1 may serve as the curve $\gamma$. Now the semianalytic set $Z=s(s(X)) \cup \bigcup_{i \in I} Z^{i}$ satisfies our requirement, since $s(s(X))$ is nowhere dense in $s(X)$.

Remark 2.1. The elimination of a semianalytic subset $Z$ of small dimension is necessary in Proposition 2.2 For example, consider the analytic subset $X$ of $\mathbb{R}^{3}$ defined by $\left(y^{2}+z^{2}\right)^{2}-4 x^{4} z^{2}=0$. Then the intersection of $X$ with the plane $x=a$ is two tangent circles with radii $a^{2}$ and tangent point $(a, 0,0)$. The set of singular points $s(X)$ is the whole $x$-axis. But for each $C^{1}$ curve starting with $(0,0,0)$ and being contained in $X$, its tangent vector at $(0,0,0)$ belongs to the $x$-axis. 


\section{Conjugacy Classes of Finite order in LiE Groups}

With the aid of the Curve Selection Lemma proved in the previous section, we can prove the main theorem of this paper now. For a Lie group $G$, denote $E_{n}(G)=\left\{g \in G \mid g^{n}=e\right\}$. First we give several lemmas.

Lemma 3.1. Let $G$ be a Lie group, $g \in G$. Let $C_{g}$ be the conjugacy class containing $g$. Then as an immersed submanifold of $G$, its tangent space at $g$ is

$$
T_{g} C_{g}=\left(d r_{g}\right)_{e}(\operatorname{Im}(1-A d(g))),
$$

where $r_{g}$ is the right translation on $G$ by $g$.

Proof. A direct computation.

Lemma 3.2. Let $G$ be a Lie group, $g \in E_{n}(G)$. Let $\gamma:[0,1) \rightarrow G$ be a $C^{1}$ curve such that $\gamma(0)=g, \gamma((0,1)) \subset E_{n}(G)$. Then

$$
\gamma^{\prime}(0) \in\left(d r_{g}\right)_{e}\left(\operatorname{ker}\left(1+A d(g)+\cdots+A d\left(g^{n-1}\right)\right)\right) .
$$

Proof. Let $\alpha(t)=\gamma(t) g^{-1}$. Then

$$
e=\gamma(t)^{n}=(\alpha(t) g)^{n}=\alpha(t)\left(g \alpha(t) g^{-1}\right) \cdots\left(g^{n-1} \alpha(t) g^{-(n-1)}\right) .
$$

Applying $\left.\frac{d}{d t}\right|_{t=0}$ two both sides of the above identity, we get

$$
\left(1+A d(g)+\cdots+A d\left(g^{n-1}\right)\right)\left(\alpha^{\prime}(0)\right)=0 .
$$

Note also that $\gamma^{\prime}(0)=\left(d r_{g}\right)_{e}\left(\alpha^{\prime}(0)\right)$, the lemma is proved.

Lemma 3.3. Let $G$ be a Lie group, $g \in E_{n}(G)$. Then

$$
\operatorname{ker}\left(1+A d(g)+\cdots+A d\left(g^{n-1}\right)\right)=\operatorname{Im}(1-A d(g))
$$

on the Lie algebra $\mathfrak{g}$ of $G$.

Proof. First we have $\left(1+A d(g)+\cdots+A d\left(g^{n-1}\right)\right) \circ(1-A d(g))=0$. So

$$
\operatorname{Im}(1-A d(g)) \subset \operatorname{ker}\left(1+A d(g)+\cdots+A d\left(g^{n-1}\right)\right) .
$$

Hence to prove the lemma, it is sufficient to show that

$$
\operatorname{rank}(1-A d(g))+\operatorname{rank}\left(1+A d(g)+\cdots+A d\left(g^{n-1}\right)\right) \geq \operatorname{dim}(\mathfrak{g}) .
$$

We claim that

$$
\operatorname{ker}(1-A d(g)) \cap \operatorname{ker}\left(1+A d(g)+\cdots+A d\left(g^{n-1}\right)\right)=\{0\} .
$$

If this is true, then the inequality about ranks follows immediately. Now we verify the claim. In fact, if $X \in \mathfrak{g}$ belongs to the left hand side of the above equality, then $(1-A d(g)) X=0$, which means that $A d(g) X=X$. So $0=(1+A d(g)+\cdots+$ $\left.A d\left(g^{n-1}\right)\right) X=n X$. This shows $X=0$. The claim is proved.

Theorem 3.4. Let $G$ be a connected Lie group, $n$ a positive integer. Then each connected component of $E_{n}(G)$ is a conjugacy class of $G$.

Proof. Endow $G$ with the unique real analytic structure such that group operations are real analytic, then $E_{n}(G)$ is an analytic subset of $G$. Let $E^{i}$ be a connected component of $E_{n}(G)$, then $E^{i}$ is semianalytic. Since $E_{n}(G)$ is invariant under the adjoint action of $G$ and $G$ is connected, $E^{i}$ is invariant under the adjoint action of $G$. Hence $r\left(E^{i}\right)$ and $s\left(E^{i}\right)$ are also invariant under the adjoint action of $G$. We claim that $s\left(E^{i}\right)=\emptyset$. For otherwise, by Proposition 2.2 there exists a $g \in s\left(E^{i}\right)$ and a 
$C^{1}$ curve $\gamma:[0,1) \rightarrow G$ such that $\gamma(0)=g, \gamma((0,1)) \subset r\left(E^{i}\right)$, and $\gamma^{\prime}(0) \notin T_{g} N$ for any analytic immersed submanifold $N$ of $G$ with $g \in N \subset s\left(E^{i}\right)$. In particular, $\gamma^{\prime}(0) \notin T_{g} C_{g}$, where $C_{g}$ is the conjugacy class containing $g$, which is an analytic immersed submanifold of $G$. But by Lemma 3.1 Lemma 3.2 and Lemma 3.3 we have $\gamma^{\prime}(0) \in T_{g} C_{g}$, a contradiction. This shows $s\left(E^{i}\right)=\emptyset$, that is, $E^{i}$ is an analytic submanifold of $G$.

The proof of Lemma 3.2 in fact shows

$$
T_{g} E^{i}=\left(d r_{g}\right)_{e}\left(\operatorname{ker}\left(1+A d(g)+\cdots+A d\left(g^{n-1}\right)\right)\right)
$$

for each $g \in E^{i}$. By Lemma 3.1 and Lemma $3.3 T_{g} E^{i}=T_{g} C_{g}$. This means that the infinitesimal transformations of $\mathfrak{g}$ on $E^{i}$ induced by the adjoint action generate $T_{g} E^{i}$ at each $g \in E^{i}$. So the adjoint action of $G$ acts transitively on $E^{i}$. In other word, we have $E^{i}=C_{g}$. The theorem is proved.

Let $n$ be a positive integer. An element $g$ in a Lie group is of order $n$ if $g^{n}=e$. A conjugacy class in a Lie group is of order $n$ if it contains an element of order $n$, in which case all elements in this conjugacy class are of order $n$. The following corollary is obvious.

Corollary 3.5. Let $G$ be a connected Lie group, then all conjugacy classes of order $n$ in $G$ are closed for each positive integer $n$.

Remark 3.1. By an argument similar to the proof of Theorem 3.4 one can easily show that for a Lie group $G$ with finite many components, each conjugacy class in $G$ of order $n$ is a union of finite many connected components of $E_{n}(G)$, which is closed. We leave the details to the reader.

The following proposition gives a description of the subsets $E_{n}(G)$.

Proposition 3.6. Let $G$ be a connected Lie group. Then $\overline{\bigcup_{n=1}^{\infty} E_{n}(G)}=\overline{\bigcup_{K \in \mathcal{K}(G)} K}$, where $\mathcal{K}(G)$ denotes the set of all maximal compact subgroups of $G$. In particular, if $G$ is compact, then $\bigcup_{n=1}^{\infty} E_{n}(G)$ is dense in $G$.

Proof. Let $n$ be a positive integer and $g \in E_{n}$. Then the set $\left\{e, g, \cdots, g^{n-1}\right\}$ is a finite (hence compact) subgroup of $G$. By Theorem 3.1 of Chapter XV in Hochschild [1, there exists a maximal compact subgroup $K$ of $G$ containing $g$. So $E_{n} \subset \bigcup_{K \in \mathcal{K}(G)} K$, and hence $\overline{\bigcup_{n=1}^{\infty} E_{n}(G)} \subset \overline{\bigcup_{K \in \mathcal{K}(G)} K}$.

Conversely, let $K$ be a maximal compact subgroup $K$ of $G$, which is necessary connected (also by the loc. cit. Theorem in 1]). Let $T$ be a maximal torus of $K$. Then it is obvious that $T=\overline{\bigcup_{n=1}^{\infty} E_{n}(T)} \subset \overline{\bigcup_{n=1}^{\infty} E_{n}(G)}$. But $K$ is the union of all maximal tori of $K$. So $K \subset \overline{\bigcup_{n=1}^{\infty} E_{n}(G)}$, and hence $\overline{\bigcup_{K \in \mathcal{K}(G)} K} \subset \overline{\bigcup_{n=1}^{\infty} E_{n}(G)}$. This proves the proposition.

\section{Some properties of connected components of $E_{n}(G)$}

In this section we present some properties of connected components of the subset $E_{n}(G)$. To simply notations, We denote the set of all connected components of $E_{n}(G)$ by $\mathcal{E}_{n}(G)$.

Corollary 4.1. Let $G$ be a connected Lie group, $n$ a positive integer. Then each element of $\mathcal{E}_{n}(G)$ is a closed submanifold of $G$. 
Proof. Let $E^{i}$ be an element of $\mathcal{E}_{n}(G)$, that is, a connected component of $E_{n}(G)$. By Theorem 3.4 $E^{i}$ is a conjugacy class in $G$, so it is an immersed submanifold of $G$. But as a connected component of $E_{n}(G), E^{i}$ is closed in $E_{n}(G)$, hence closed in $G$. This proves $E^{i}$ is a closed submanifold of $G$.

It is obvious that $E_{n}(G) \cap E_{m}(G)=E_{(n, m)}(G)$, where $(n, m)$ is the greatest common divisor of $n$ and $m$. But without the help of Theorem 3.4 the following conclusion is not so obvious.

Corollary 4.2. Let $G$ be a connected Lie group, and let $n, m$ be positive integers. Then $\mathcal{E}_{n}(G) \cap \mathcal{E}_{m}(G)=\mathcal{E}_{(n, m)}(G)$. In particular, if $n$ divides $m$, then $\mathcal{E}_{n}(G) \subset$ $\mathcal{E}_{m}(G)$.

Proof. Suppose $E^{i} \in \mathcal{E}_{(n, m)}(G)$. By Theorem 3.4 $E^{i}$ is a conjugacy class. Note that $E^{i} \subset E_{n}(G)$, also by Theorem 3.4. $E^{i} \in \mathcal{E}_{n}(G)$. Similarly, $E^{i} \in \mathcal{E}_{m}(G)$. So we have $\mathcal{E}_{(n, m)}(G) \subset \mathcal{E}_{n}(G) \cap \mathcal{E}_{m}(G)$.

Conversely, suppose $E^{j} \in \mathcal{E}_{n}(G) \cap \mathcal{E}_{m}(G)$. Then $E^{j}$ is a conjugacy class contained in $E_{(n, m)}(G)$, and then is an element of $\mathcal{E}_{(n, m)}(G)$. So we also have $\mathcal{E}_{n}(G) \cap \mathcal{E}_{m}(G) \subset$ $\mathcal{E}_{(n, m)}(G)$. This proves the corollary.

Remark 4.1. Since $E_{n}(G) \cap E_{m}(G)=E_{(n, m)}(G)$, Corollary 4.2 in fact says $\mathcal{E}_{n}(G) \cap$ $\mathcal{E}_{m}(G)$ coincides with the set of all connected components of $E_{n}(G) \cap E_{m}(G)$.

Let $H$ be another Lie group and let $f: H \rightarrow G$ be a homomorphism of Lie groups. Then $f$ induces a map $f_{*}: \mathcal{E}_{n}(H) \rightarrow \mathcal{E}_{n}(G)$ in the natural way. In the case that $K$ is a maximal compact subgroup of $G$ and $i: K \rightarrow G$ is the inclusion map, the induced map $i_{*}: \mathcal{E}_{n}(K) \rightarrow \mathcal{E}_{n}(G)$ is a bijection. To prove this, we need the following lemma.

Lemma 4.3. Let $G$ be a connected Lie group, $K$ a maximal compact subgroup of $G$. Then two elements of $K$ are conjugate in $K$ if and only if they are conjugate in $G$.

Proof. The "only if" part is obvious. We prove the "if" part.

By Theorem 3.1 of Chapter XV in [1], there exist some linear subspaces $\mathfrak{m}_{1}, \cdots, \mathfrak{m}_{r}$ of the Lie algebra $\mathfrak{g}$ of $G$ such that

(1) $\mathfrak{g}=\mathfrak{k} \oplus \mathfrak{m}_{1} \oplus \cdots \oplus \mathfrak{m}_{r}$, where $\mathfrak{k}$ is the Lie algebra of $K$;

(2) $\operatorname{Ad}(k)\left(\mathfrak{m}_{i}\right)=\mathfrak{m}_{i}, \forall k \in K, i \in\{1, \cdots, r\}$;

(3) the map $\varphi: K \times \mathfrak{m}_{1} \times \cdots \times \mathfrak{m}_{r} \rightarrow G$ defined by $\varphi\left(k, X_{1}, \cdots, X_{r}\right)=k e^{X_{1}} \cdots e^{X_{r}}$ is a diffeomorphism.

Now suppose $k_{1}, k_{2} \in K$ are conjugate in $G$, that is, there exists some $g \in G$ such that $k_{2}=g k_{1} g^{-1}$. Rewrite this equality as $k_{1}^{-1} g k_{1}=k_{1}^{-1} k_{2} g$, and write $g=k e^{X_{1}} \cdots e^{X_{r}}$, where $k \in K, X_{i} \in \mathfrak{m}_{i}$. Then we have

$$
\left(k_{1}^{-1} k k_{1}\right) e^{A d\left(k_{1}^{-1}\right) X_{1}} \cdots e^{\operatorname{Ad}\left(k_{1}^{-1}\right) X_{r}}=\left(k_{1}^{-1} k_{2} k\right) e^{X_{1}} \cdots e^{X_{r}},
$$

that is,

$$
\varphi\left(k_{1}^{-1} k k_{1}, \operatorname{Ad}\left(k_{1}^{-1}\right) X_{1}, \cdots, A d\left(k_{1}^{-1}\right) X_{r}\right)=\varphi\left(k_{1}^{-1} k_{2} k, X_{1}, \cdots, X_{r}\right) .
$$

Since $\varphi$ is a diffeomorphism, we have $k_{1}^{-1} k k_{1}=k_{1}^{-1} k_{2} k$, that is, $k_{2}=k k_{1} k^{-1}$. Hence $k_{1}, k_{2}$ are conjugate in $K$.

Corollary 4.4. Let $G$ be a connected Lie group, $K$ a maximal compact subgroup of $G$. Then $i_{*}: \mathcal{E}_{n}(K) \rightarrow \mathcal{E}_{n}(G)$ is a bijection. 
Proof. Suppose $E^{i} \in \mathcal{E}_{n}(G)$, and choose an arbitrary element $g \in E^{i}$. By Theorem 3.1 of Chapter XV in [1], there exists $h \in G$ such that $h g h^{-1} \in K$. But $E^{i}$ is a conjugacy class in $G$, so $h g h^{-1} \in E^{i}$. This proves $E^{i} \cap K \neq \emptyset$. Hence $i_{*}$ is surjective.

The above argument in fact shows $E^{i} \cap K$ is a non-empty union of some elements of $\mathcal{E}_{n}(K)$ for each $E^{i}$. We claim that such a union consists of just one element of $\mathcal{E}_{n}(K)$. In fact, if $E_{K}^{1}, E_{K}^{2} \in \mathcal{E}_{n}(K)$ such that $E_{K}^{1} \cup E_{K}^{2} \subset E^{i} \cap K$, choose $k_{1} \in E_{K}^{1}, k_{2} \in E_{K}^{2}$. Since $k_{1}$ and $k_{2}$ belong to $K^{i}$, they are conjugate in $G$. By Lemma 4.3 they are conjugate in $K$. But $E_{K}^{1}, E_{K}^{2}$ are conjugacy classes in $K$, this forces $E_{K}^{1}=E_{K}^{2}$. In other word, the map $i_{*}$ is injective.

The following conclusion is obvious from the proof the Corollary 4.4

Corollary 4.5. Let $G$ be a connected Lie group, $K$ a maximal compact subgroup of $G$. Then the intersection of each element of $\mathcal{E}_{n}(G)$ with $K$ is an elememt of $\mathcal{E}_{n}(K)$.

Corollary 4.6. Let $G$ be a connected Lie group. Then $\mathcal{E}_{n}(G)$ is a finite set.

Proof. Let $K$ be a maximal compact subgroup of $G$, and let $T$ be a maximal torus of $K$. By the theory of compact Lie groups, the natural map $\mathcal{E}_{n}(T) \rightarrow \mathcal{E}_{n}(K)$ is surjective. But $\mathcal{E}_{n}(T)$ is obvious finite, so $\mathcal{E}_{n}(K)$ is also finite. By Corollary 4.4 $\mathcal{E}_{n}(G)$ and $\mathcal{E}_{n}(K)$ have the same cardinalities. So $\mathcal{E}_{n}(G)$ is finite.

\section{ACKNOWLEDGMENTS}

The first author would like to thank the following people: Professor Z. Hajto and M. Shiota for valuable conversations, Professor Jiu-Kang Yu for helpful discussion on algebraic groups and for pointing out some errors in the first draft of the paper, Professor K.-H. Neeb for informing the author a Lie-theoretic proof of Theorem 1.1 after the first draft of this paper was available, as well as Dong Wang and Kuihua Yan for friendly help.

\section{REFERENCES}

[1] Hochschild, G., The structrue of Lie groups, Holden-Day, San Francisco, 1965.

[2] Humphreys, J., Conjugacy classes in semisimple algebraic groups, American Mathematical Society, Providence, RI, 1995.

[3] Łojasiewicz, S., Ensembles semi-analytiques, preprint, IHES, 1965.

[4] Varadarajan, V. S., Lie groups, Lie algebras, and their representations, Springer-Verlag, New York/Berlin, 1984.

[5] Wall, C. T. C., Regular stratifications, in Dynamical systems-Warwick 1974, 332-344, Lecture Notes in Math., Vol. 468, Springer, Berlin, 1975.

School of mathematical science, Peking University, Beijing, 100871, P. R. China

E-mail address: anjinpeng@pku.edu.cn

School of mathematical science, Peking University, Beijing, 100871, P. R. China

E-mail address: zdwang@pku.edu.cn 